\title{
Prevalence, co-morbidity and correlates of mental disorders in the general population: results from the German Health Interview and Examination Survey (GHS)
}

\author{
F. JACOBI*, H.-U. WITTCHEN, C. HÖLTING, M. HÖFLER, H. PFISTER, \\ N. MÜLLER AND R. LIEB \\ Institute of Clinical Psychology and Psychotherapy, Unit: Epidemiology and Service Research, \\ Technical University of Dresden, Germany; Max Planck Institute of Psychiatry, Clinical Psychology \\ and Epidemiology, Munich, Germany
}

\begin{abstract}
Background. The German National Health Interview and Examination Survey (GHS) is the first government mandated nationwide study to investigate jointly the prevalence of somatic and mental disorders within one study in the general adult population in Germany. This paper reports results from its Mental Health Supplement (GHS-MHS) on 4-week 12-month, and selected lifetime prevalence of a broad range of DSM-IV mental disorders, their co-morbidity and correlates in the community.
\end{abstract}

Methods. The sample of the GHS-MHS $(n=4181$; multistage stratified random sample drawn from population registries; conditional response rate: $87.6 \%$ ) can be regarded as representative for the German population aged 18-65. Diagnoses are based on fully structured computer assisted clinical interviews (M-CIDI), conducted by clinically trained interviewers.

Results. 12-month prevalence for any DSM-IV study disorder is 31\% (lifetime: 43\%; 4-week: $20 \%$ ) with anxiety disorders, mood disorders and somatoform syndromes being the most frequent diagnoses. Retrospective age of onset information reveals that most disorders begin early in life. Comorbidity rates among mental disorders range from $44 \%$ to $94 \%$. Correlates of increased rates of mental disorders and co-morbidity were: female gender (except for substance disorders), not being married, low social class, and poor somatic health status. Health care utilization for mental disorders depended on co-morbidity (30\% in 'pure', $76 \%$ in highly co-morbid cases) and varied from $33 \%$ for substance use disorders to $75 \%$ for panic disorder.

Conclusions. Results confirm and extend results from other national studies using the same assessment instruments with regard to prevalence, co-morbidity and sociodemographic correlates, covering a broader range of DSM-IV disorders [i.e. somatoform disorders, all anxiety disorders (except PTSD), mental disorders due to substance or general medical factor, eating disorders]. Intervention rates were higher than in previous studies, yet still low overall.

\section{INTRODUCTION}

Historically, despite some notable regional exceptions (e.g. Iceland; Hagnell, 1970), there

\footnotetext{
* Address for correspondence: Dr Frank Jacobi, TU Dresden, Klinische Psychologie und Psychotherapie, Chemnitzer Str. 46, D-01187 Dresden, Germany.
}

have been only few nationally representative community surveys on the prevalence of mental disorders before the 1980s and those available revealed tremendous variation in findings (Weissman et al. 1993). This has been explained by various factors, including: the misconception that mental disorders are fairly infrequent 
phenomena, the lack of reliable diagnostic criteria and diagnostic instruments, the almost exclusive focus on broad diagnostic categories of severe psychotic and neurotic disorders, the lack of efficient treatments and the lack of a broader spectrum of mental health services. Parallel to the introduction of explicit diagnostic criteria for specific forms of mental disorders in the DSM-III (APA, 1980), and subsequent to the landmark Epidemiological Catchment Area study (ECA; Regier et al. 1984; Robins \& Regier, 1990) in 1980, this situation changed considerably. The ECA not only demonstrated that mental disorders can be assessed using standardized diagnostic interviews with a similar level of reliability, validity and accuracy as the assessment in surveys of somatic disorders, but also suggested that the risk of developing mental disorders in adolescence and over a lifetime, as well as the current prevalence and co-morbidity had been heavily underestimated.

Continuing interest in mental disorders has prompted the conduct of numerous nationwide mental health surveys, which have shown fairly convergently and with increasing sophistication that mental disorders affect at least one third of population over their lifetime and at least about one fifth in the past month. Examples include the Munich Follow-up Study in former West Germany (Wittchen, 1988), subsequent reanalyses of the Cross-National Collaborative Group (e.g. Weissman et al. 1996), the National Comorbidity Survey in the USA (NCS; Kessler et al. 1994), the Australian National Mental Health Survey (ANMHS; Andrews et al. 2001), the National Psychiatric Morbidity Surveys of Great Britain (NPMS; Jenkins et al. 1997), The Netherlands Mental Health Survey and Incidence Study (NEMESIS; Bijl et al. 1998), and more recently several other countries around the world that have been involved in the cross national comparative studies of the WHO International Consortium in Psychiatric Epidemiology (Andrade et al. 2000).

Despite these developments, numerous critical and unresolved issues still make it difficult to draw firm conclusions about the size and scope of mental disorders, their associated correlates and consequences which could potentially provide guidance for health care planning. These include the following considerations.
(1) Even though the majority of epidemiological studies made use of the same diagnostic instrument to obtain diagnoses according to the criteria of DSM-III-R and IV (APA, 1987, 1994), the Diagnostic Interview Schedule (DIS; Robins et al. 1981) and its successor, the Composite International Diagnostic Interview (CIDI; WHO, 1997), there is still substantial and sometimes confusing variation in findings (even more when different diagnostic instruments are used).

(2) Some variations can be explained by design issues: the prevalence of mental disorders has been shown to differ by age groups and age cohort, thus the year the study was conducted and the age range of the sample is of relevance.

(3) Almost all studies made at least some modification in their assessment instrument, by either adding diagnoses, omitting diagnoses, changing diagnostic thresholds or algorithms, changing the order of sections, adding questions on impairment and help-seeking or dimensional measures; other important sources of variance might result from the use of lay or alternatively clinical interviewers. All of these modifications have been shown to have potentially significant effects on prevalence estimates as well as comorbidity figures (Brugha et al. 1999; Wittchen et al. 1999 a).

(4) A critical issue for health care policy decisions and implications derived from such studies is either lack or inconsistency of evaluation of disability and severity as well as helpseeking behaviour associated with mental disorders. Such considerations are of major importance for health care planners to determine met and unmet needs appropriately for different target groups. This problem is particularly evident in prevalence estimates of studies based on the lifetime version of the DIS or CIDI. The lifetime version of the CIDI ascertains primarily whether the diagnostic criteria are met at some point in the respondent's life and then asks for the first (onset) and last occurrence (recency codes) of at least some of the core features of the respective diagnosis. In these studies, current prevalence estimates merely indicate that the person had the diagnosis in the past and still has some symptoms without necessarily determining that the person meets the full set of diagnostic criteria. For health care purposes and need estimations, however, this type of diagnostic 
cross-sectional information is crucial, especially in conjunction with information on associated impairment, disability and help-seeking behaviour in order to make an appropriate evaluation of need.

Against this background, in 1997 the German government commissioned a comprehensive nationwide morbidity and health survey in the community to describe in a coordinated study the prevalence of somatic and mental disorders in the adult German population. This project is unique in two respects: it is the first nationwide mental health survey in Germany and also the first survey in which somatic and mental disorders were both rigorously assessed within one study, using reliable structured somatic and psychiatric interviews in a representative national sample. Particular requirements for the mental health survey were: (1) a broad coverage of specific mental disorders, including all substance use and anxiety disorders (except PTSD) and previously neglected disorders like somatoform and eating disorders, as well as disorders due to substance and general medical factors, (2) the provision of predominantly strict 12-month estimates for symptoms and diagnoses of mental disorders in order to allow for the examination of associations with physical morbidities assessed for the same time frame and to make comparative analyses between disorders with regard to disabilities, quality of life and health service utilization.

This paper reports the basic prevalence findings of the GHS-MHS, focusing on four aims: (1) to report 12-month and selected 1-month and lifetime prevalence estimates and associated correlates of mental disorders in a nationally representative sample, including information on ages of onset; (2) to determine the degree of co-morbidity between mental disorders; (3) to determine associations between mental disorders, co-morbidity and treatment utilization, and (4) to report associated correlates of disorders and co-morbidity.

\section{METHODS}

Aims, design and methods have been recently described in greater detail in a separate publication (Jacobi et al. 2002). Therefore, design and sample characteristics are discussed only briefly here.

\section{Design and sample}

Mental disorders were assessed in the Mental Health Supplement of the German National Health Interview and Examination Survey (GHS-MHS) in a subsample of its core survey (GHS-CS; Bellach et al. 1998). The core survey covered a range of medical and social assessments and was administered between June 1998 and October 1999. Its sample was a stratified random sample from 113 communities throughout Germany with 130 sampling units (sampling steps: 1, selection of communities; 2, selection of sampling units; and 3, selection of inhabitants). The sample was drawn from the population registries of subjects aged 18-79 living in Germany in the year 1997. As a result a gross sample of 13222 people were eligible, representative according to the age, sex and community type criteria. The response rate (completing the total assessment in the GHSCS) was $61.4 \%(n=7124)$. The response rate including subjects completing parts of the assessment was $77 \cdot 8 \%$. Reasons for nonparticipation, analyses of non-response, and further information on sample and weighting in the GHS-CS are provided elsewhere (Thefeld et al. 1999).

For financial and logistical reasons the data for mental disorders were gathered using a twostage design. The first stage entailed the administration of a 12-item screening questionnaire for mental disorders at the end of the medical examination of the core survey [CID-S; sensitivity for any 12-month diagnosis ('caseness'): $85.3 \%$, specificity: $55.3 \%$, positive predictive value: $38.3 \%$, negative predictive value: $92.0 \%$; Wittchen et al. 1999 b].

The second stage involved the separate administration of a complete, structured, clinical psychopathological interview to all core survey respondents who had been screened positive for a mental disorder and to a random sample of $50 \%$ who had been screened negative. Due to the resulting oversampling of screen positives, data were weighted in the later analyses. In order to adjust the net sample to German age, sex and community distribution, data were also weighted for age, sex, and region corresponding to the national administrative statistics of December 1997. The presented weighted results can be regarded as representative for the German 
non-instutionalized adult population from 18 to 65 years of age with sufficient language skills to follow the interviews. Hospitalized patients who were hospitalized throughout the recruitment phase $(0 \cdot 5-0.8 \%$ of the target population in the sample points) were only omitted if they were in institutions during the entire recruitment period. Therefore it is unlikely that this exclusion criterion might have an effect on the reported prevalences. Respondents of the German Health Survey older than 65 years were excluded because the psychometric properties of the CIDI, the interview used in the study, have not yet been satisfactorily established for use in older populations (Knäuper \& Wittchen, 1994).

After exclusion of the subjects older than 65 and $50 \%$ of the screen negatives, the eligible sample size for the GHS-MHS was $n=4773$. The conditional response rate of the GHS-MHS was $87.6 \%$, resulting in a total of 4181 respondents who completed the mental health assessment. Sociodemographic variables of the sample are shown in the first column of Table 3.

\section{Assessment}

We present DSM-IV prevalences because (1) their operationalization in terms of criteria is more straightforward and (2) the validity of ICD-10 diagnostic criteria for research (ICD10-DCR) has never been established for the CIDI. ICD-10 compatible codes can easily be derived from the coding rules for 'translation' from DSM-IV.

Psychopathological and diagnostic assessments were based on the computer-assisted version of the Munich Composite International Diagnostic Interview (DIA-X/M-CIDI; Wittchen \& Pfister, 1997), a modified version of the World Health Organization CIDI (version 2.1 ; WHO, 1997) for a wider range of mental disorder according to the criteria of DSM-IV than previous studies.

Unlike previous versions of the CIDI, the study version focuses strictly on the assessment of 12-month symptoms and disorders. The standard CIDI lifetime assessment was only performed when lifetime information was necessary for evaluating current diagnoses (e.g. mood disorders).

Psychometric properties of the CIDI were found to range from acceptable to very good
(Wittchen, 1994; Lachner et al. 1998; Reed et al. 1998). The interviewers $(n=24$, mostly psychologists who had already worked on other CIDI studies) had received a 3-day CIDI training session for the GHS-MHS and CIDI refresher courses every 3 months throughout the field period. They conducted on the average 174 interviews in eight sampling units and were closely monitored and provided with feedback by trained M-CIDI clinical editors who regularly checked all interviewers according to a standard procedure. In a final quality control only eight interviews had to be eliminated due to missing or inconsistent datasets (Jacobi et al. 2002).

The following mental disorders according to DSM-IV were included: mental disorders due to general medical condition; schizophrenia and possible psychotic disorders (screening without further differential diagnosis); substance use disorders (dependence and abuse of alcohol, illicit substances, and nicotine); mood disorders (unipolar and bipolar); anxiety disorders (including obsessive compulsive disorder; without PTSD), somatoform disorders (including the abridged somatization syndrome SSI4.6; Escobar et al. 1989; without conversion and body dysmorphic disorder); and eating disorders. Unfortunately, we were not commissioned to include post-traumatic stress disorder and antisocial personality (as for example in the NCS) because of time and financial restrictions. Subthreshold diagnoses could be assessed as well (Carter et al. 2001). Health care utilization is presented in form of the variable 'at least minimal intervention', a combination of items asking about having ever sought treatment due to psychological, mental, addictive or psychosomatic problems, or been recommended by a doctor to do so.

\section{Analytic strategy}

Presented prevalence estimates $(n, \%$, S.E.) were calculated with the data weighted for age, gender, region and screening status in order to address different sampling probabilities and systematic non-response. Logistic regression (odds ratios and 95\% confidence intervals) were used to quantify the associations between mental disorders and their correlates.

Age and sex were adjusted for in each analysis to control for different base distributions of 
prevalence and co-morbidity within the sample. To account for the weighting scheme as well as the stratified sampling design by screening status, statistical inference (standard errors, confidence intervals and $p$ values) was based on the Huber-White sandwich estimator of variance (Woodruff, 1971; Binder, 1983; Royall, 1986). This was done with the Stata software package, release 7.0 (StataCorp, 2001).

\section{RESULTS}

Table 1 shows CIDI/DSM-IV prevalence estimates and corresponding standard errors of a range of selected lifetime, 12-month, and 4-week disorders (or aggregated diagnostic categories) assessed in the GHS-MHS.

\section{Overall prevalences}

About $20 \%$ of the sample reported at least one of the listed disorders in Table 1 in the past 4 weeks, $31 \%$ had one or more disorders in the 12 months before the interview and a total of $43 \%$ had a lifetime history of at least one of the selected DSM-IV disorders.

\section{Diagnostic groups}

As an aggregated category, anxiety disorders were more prevalent (4-week and 12-month, lifetime not assessed) than somatoform disorders/ syndromes and mood disorders which show comparable prevalence numbers: in the previous 12 months one in every seven respondents had fulfilled the criteria for an anxiety disorder, and about one of every 10 respondents had suffered from a mood or somatoform disorder/ syndrome. Substance use disorders (without nicotine) in the previous 12 months were diagnosed in about one in every 20 participants.

A considerable number met criteria of the M-CIDI psychosis screen (4-week: $1.5 \%$; 12-month: $2 \cdot 6 \%$; lifetime: $4 \cdot 5 \%$ ). This count includes about equal proportions of subjects with mood congruent or incongruent psychotic features occurring exclusively in the course of depressive or bipolar disorders, and subjects reporting psychotic symptoms of varying duration occurring at other times. M-CIDI syndrome information for psychotic disorders do not allow for the derivation of specific diagnoses of psychotic disorders such as schizophrenia.
The least prevalent diagnoses were mental disorders due to a general medical condition (4-week: $0.5 \% ; 12$-month: $1.3 \%$ ) and eating disorders (4-week : $0 \cdot 2 \% ; 12$-month: $0 \cdot 3 \%$ ).

\section{(Aggregated) subdiagnoses}

The most common mental disorders were phobic disorders (4-week: 7.4\% ; 12-month: 12.6\%; lifetime not assessed), depressive disorders (4-week: $5 \cdot 6 \%$; 12-month: $10.7 \%$; lifetime: $17 \cdot 1 \%$ ), and somatoform pain disorder (4-week: $5 \cdot 4 \% ; 12$-month: $8 \cdot 1 \%$; lifetime: $12 \cdot 7 \%$ ).

The next most common disorders were alcohol abuse or dependence (4-week: $2 \cdot 5 \%$; 12-month: $4 \cdot 1 \%$; lifetime: $8.5 \%$ ) and the subthreshold somatization disorder SSI4.6 (Escobar et al. 1989; 4-week: 3.1\% ; 12-month: $4.3 \%$; lifetime: $5 \cdot 6 \%$ ).

\section{Gender differences}

As in previous epidemiological studies men fulfil diagnostic criteria for mental disorders less often than women. The only exceptions are substance use disorders, where men have considerably higher prevalence rates than women (especially alcohol abuse or dependence), and possible psychotic disorders (distributed equally among men and women). In addition, women seem more likely than men to suffer from comorbid mental disorders (4-week: 40.3\% v. $30.3 \%$; 12 -month: $43.7 \%$ v. $33.5 \%$; lifetime: $48 \cdot 7 \%$ v. $35 \cdot 7 \%$; not shown in Table 1 ).

\section{Ages of onset}

In the GHS-MHS, ages of onset were assessed retrospectively. Figure 1 shows the cumulative distribution of age of onset for selected disorders [note: for anxiety disorders (except panic disorders) ages of onset were only assessed in subjects with a 12-month diagnosis].

Most mental disorders develop in adolescence and early adulthood, reaching the median of lifetime disorders by the age of 20 (anxiety, bipolar mood, somatoform and substance use disorders). Depressive disorders and possible psychotic disorders tend to emerge later $($ median $=31$ years $/ 37$ years). The range between the 25 th and the 75th percentile of the age of onset is lowest in substance and bipolar disorders, where over $75 \%$ begin before the age of 30 and prevalence drops sharply in respondents older than 30 . 
Table 1. Prevalences of mental disorders (4-week, 12-month and lifetime M-CIDI/DSM-IV) in the general population $(G H S-M H S ; n=4181)^{\mathrm{a}}$

\begin{tabular}{|c|c|c|c|c|c|c|c|c|c|c|c|c|}
\hline \multirow[b]{3}{*}{ Disorders (DSM-IV) } & \multicolumn{4}{|c|}{ 4-week } & \multicolumn{4}{|c|}{ 12-month } & \multicolumn{4}{|c|}{ Lifetime } \\
\hline & \multicolumn{2}{|c|}{ Total } & \multirow{2}{*}{$\begin{array}{l}\text { Male } \\
\% \text { (S.E.) }\end{array}$} & \multirow{2}{*}{$\begin{array}{l}\text { Female } \\
\% \text { (s.E.) }\end{array}$} & \multicolumn{2}{|c|}{ Total } & \multirow{2}{*}{$\begin{array}{c}\text { Male } \\
\% \text { (S.E.) }\end{array}$} & \multirow{2}{*}{$\begin{array}{l}\text { Female } \\
\% \text { (s.E.) }\end{array}$} & \multicolumn{2}{|r|}{ Total } & \multirow{2}{*}{$\begin{array}{c}\text { Male } \\
\% \text { (s.E.) }\end{array}$} & \multirow{2}{*}{$\begin{array}{l}\text { Female } \\
\% \text { (S.E.) }\end{array}$} \\
\hline & $n^{\mathrm{j}}$ & $\%(\text { S.E. })^{\mathrm{k}}$ & & & $n$ & $\%$ (S.E.) & & & $n$ & $\%$ (S.E.) & & \\
\hline Any mental disorder due to general medical condition & 19 & $0 \cdot 5(0 \cdot 1)$ & $0 \cdot 4(0 \cdot 1)$ & $0 \cdot 6(0 \cdot 1)$ & 56 & $1 \cdot 3(0 \cdot 2)$ & $0 \cdot 8(0 \cdot 2)$ & $1.9(0 \cdot 3)$ & 94 & $2 \cdot 3(0 \cdot 2)$ & $1 \cdot 8(0 \cdot 3)$ & $2 \cdot 7(0 \cdot 4)$ \\
\hline Any substance disorder ${ }^{\mathrm{b}}$ & 119 & $2 \cdot 9(0 \cdot 3)$ & $4 \cdot 4(0 \cdot 5)$ & $1 \cdot 3(0 \cdot 3)$ & 188 & $4 \cdot 5(0 \cdot 3)$ & $7 \cdot 2(0 \cdot 6)$ & $1 \cdot 7(0 \cdot 2)$ & 414 & $9 \cdot 9(0 \cdot 6)$ & $15 \cdot 6(0 \cdot 9)$ & $4 \cdot 2(0 \cdot 4)$ \\
\hline Alcohol abuse/dependence & 104 & $2 \cdot 5(0 \cdot 3)$ & $4 \cdot 0(0 \cdot 5)$ & $0 \cdot 9(0 \cdot 2)$ & 170 & $4 \cdot 1(0 \cdot 3)$ & $6 \cdot 8(0 \cdot 6)$ & $1 \cdot 3(0 \cdot 2)$ & 355 & $8 \cdot 5(0 \cdot 5)$ & $14 \cdot 4(0 \cdot 9)$ & $2 \cdot 6(0 \cdot 3)$ \\
\hline Any illicit substance abuse/dependence & 21 & $0 \cdot 5(0 \cdot 1)$ & $0 \cdot 6(0 \cdot 2)$ & $0 \cdot 4(0 \cdot 1)$ & 30 & $0 \cdot 7(0 \cdot 1)$ & $1 \cdot 0(0 \cdot 2)$ & $0 \cdot 5(0 \cdot 1)$ & 90 & $2 \cdot 1(0 \cdot 2)$ & $2 \cdot 3(0 \cdot 3)$ & $1.9(0 \cdot 3)$ \\
\hline Possible psychotic disorder ${ }^{\mathrm{c}}$ & 61 & $1 \cdot 5(0 \cdot 2)$ & $1 \cdot 6(0 \cdot 3)$ & $1 \cdot 3(0 \cdot 2)$ & 107 & $2 \cdot 6(0 \cdot 3)$ & $2 \cdot 6(0 \cdot 4)$ & $2 \cdot 5(0 \cdot 3)$ & 189 & $4 \cdot 5(0 \cdot 4)$ & $4 \cdot 1(0 \cdot 5)$ & $5 \cdot 0(0 \cdot 5)$ \\
\hline Any mood disorder ${ }^{\mathrm{d}}$ & 262 & $6 \cdot 3(0 \cdot 4)$ & $4 \cdot 8(0 \cdot 5)$ & $7 \cdot 8(0 \cdot 6)$ & 499 & $11.9(0 \cdot 5)$ & $8 \cdot 5(0 \cdot 7)$ & $15 \cdot 4(0 \cdot 8)$ & 779 & $18 \cdot 6(0 \cdot 6)$ & $12 \cdot 3(0 \cdot 8)$ & $25 \cdot 0(1 \cdot 0)$ \\
\hline Any unipolar depression & 223 & $5 \cdot 6(0 \cdot 4)$ & $4 \cdot 2(0 \cdot 5)$ & $6.9(0 \cdot 6)$ & 448 & $10 \cdot 7(0 \cdot 5)$ & $7 \cdot 5(0 \cdot 6)$ & $14 \cdot 0(0 \cdot 8)$ & 716 & $17 \cdot 1(0 \cdot 6)$ & $11 \cdot 1(0 \cdot 8)$ & $23 \cdot 3(0 \cdot 9)$ \\
\hline Any bipolar disorder & 23 & $0 \cdot 6(0 \cdot 1)$ & $0 \cdot 3(0 \cdot 1)$ & $0.8(0 \cdot 2)$ & 34 & $0 \cdot 8(0 \cdot 1)$ & $0 \cdot 6(0 \cdot 1)$ & $1 \cdot 1(0 \cdot 2)$ & 42 & $1 \cdot 0(0 \cdot 1)$ & $0 \cdot 8(0 \cdot 2)$ & $1 \cdot 2(0 \cdot 2)$ \\
\hline Any anxiety disorder ${ }^{\mathrm{e}}$ & 375 & $9 \cdot 0(0 \cdot 4)$ & $5 \cdot 3(0 \cdot 5)$ & $12 \cdot 7(0 \cdot 7)$ & 604 & $14 \cdot 5(0 \cdot 5)$ & $9 \cdot 2(0 \cdot 6)$ & $19 \cdot 8(0 \cdot 9)$ & & & & \\
\hline Panic disorder ${ }^{\mathrm{f}}$ & 47 & $1 \cdot 1(0 \cdot 2)$ & $0 \cdot 9(0 \cdot 2)$ & $1 \cdot 3(0 \cdot 2)$ & 98 & $2 \cdot 3(0 \cdot 2)$ & $1 \cdot 7(0 \cdot 2)$ & $3.0(0 \cdot 4)$ & 162 & $3 \cdot 9(0 \cdot 3)$ & $2 \cdot 2(0 \cdot 3)$ & $5 \cdot 5(0 \cdot 5)$ \\
\hline Any phobia ${ }^{\mathrm{g}}$ & 311 & $7 \cdot 4(0 \cdot 4)$ & $3.9(0 \cdot 4)$ & $11.0(0 \cdot 7)$ & 525 & $12 \cdot 6(0 \cdot 6)$ & $7 \cdot 5(0 \cdot 6)$ & $17 \cdot 7(1 \cdot 0)$ & & & & \\
\hline Generalized anxiety disorder & 50 & $1 \cdot 2(0 \cdot 2)$ & $0 \cdot 9(0 \cdot 2)$ & $1 \cdot 5(0 \cdot 3)$ & 64 & $1 \cdot 5(0 \cdot 2)$ & $1 \cdot 0(0 \cdot 2)$ & $2 \cdot 1(0 \cdot 3)$ & & & & \\
\hline Obsessive compulsive disorder & 18 & $0.4(0 \cdot 1)$ & $0 \cdot 2(0 \cdot 2)$ & $0.6(0.0)$ & 30 & $0 \cdot 7(0 \cdot 1)$ & $0.6(0 \cdot 2)$ & $0.9(0 \cdot 2)$ & & & & \\
\hline Any somatoform disorder/syndrome $\mathrm{e}^{\mathrm{h}}$ & 311 & $7 \cdot 5(0 \cdot 4)$ & $4.9(0.5)$ & $10 \cdot 0(0 \cdot 7)$ & 461 & $11 \cdot 0(0 \cdot 6)$ & $7 \cdot 1(0 \cdot 6)$ & $15 \cdot 0(0 \cdot 8)$ & 678 & $16 \cdot 2(0 \cdot 7)$ & $10 \cdot 3(0 \cdot 8)$ & $22 \cdot 2(1 \cdot 0)$ \\
\hline SSI4.6 & 128 & $3 \cdot 1(0 \cdot 3)$ & $2 \cdot 2(0 \cdot 3)$ & $3.9(0.5)$ & 181 & $4 \cdot 3(0 \cdot 3)$ & $3 \cdot 1(0 \cdot 4)$ & $5 \cdot 5(0 \cdot 6)$ & 235 & $5 \cdot 6(0 \cdot 4)$ & $3 \cdot 9(0 \cdot 4)$ & $7 \cdot 3(0 \cdot 6)$ \\
\hline Pain disorder & 227 & $5 \cdot 4(0 \cdot 4)$ & $3.4(0.4)$ & $7 \cdot 5(0 \cdot 6)$ & 340 & $8 \cdot 1(0 \cdot 5)$ & $4.9(0.5)$ & $11.4(0.7)$ & 533 & $12 \cdot 7(0 \cdot 5)$ & $7 \cdot 8(0 \cdot 6)$ & $17 \cdot 8(0 \cdot 9)$ \\
\hline Any eating disorder ${ }^{i}$ & 8 & $0.2(0.0)$ & $0 \cdot 1(0 \cdot 0)$ & $0 \cdot 3(0 \cdot 1)$ & 14 & $0.3(0 \cdot 1)$ & $0 \cdot 2(0 \cdot 1)$ & $0.5(0.2)$ & 33 & $0 \cdot 8(0 \cdot 1)$ & $0 \cdot 3(0 \cdot 1)$ & $1 \cdot 3(0 \cdot 3)$ \\
\hline Any of the above & 829 & $19 \cdot 8(0 \cdot 6)$ & $15 \cdot 8(0 \cdot 9)$ & $23 \cdot 9(1 \cdot 0)$ & 1301 & $31 \cdot 1(0 \cdot 8)$ & $25 \cdot 3(1 \cdot 1)$ & $37 \cdot 0(1 \cdot 1)$ & 1791 & $42 \cdot 6(0 \cdot 8)$ & $36 \cdot 8(1 \cdot 2)$ & $48 \cdot 9(1 \cdot 1)$ \\
\hline
\end{tabular}

a Weighted data; DSM-IV hierarchy rules were dropped; age distributions and confidence intervals available on request.

b Abuse or dependence (without nicotine).

c Screening for Schizophrenia and other psychotic disorders without further differential diagnosis; includes psychotic features occurring as part of mood disorders.

d Major Depressive Disorder, Dysthymic Disorder, Bipolar I Disorders, Bipolar II Disorders, single hypomanic episode; unipolar and bipolar mood disorders do not add up to $100 \%$ because single hypomanic episode was not included in either category.

e For anxiety disorders (including Obsessive Compulsive Disorder, excluding Post-traumatic Stress Disorder) no lifetime diagnoses except for panic disorders available.

f With or without Agoraphobia.

g Agoraphobia without history of Panic Disorder, Social Phobia, Specific Phobias (animal, natural environment, blood-injection-injury, and situational type), Anxiety Disorder NOS

h Somatization Disorder, Undifferentiated Somatization Disorder, Somatic Symptom Index SSI4.6, Hypochondriasis, Pain Disorder.

Anorexia Nervosa, Atypical Anorexia Nervosa, Bulimia Nervosa, Atypical Bulimia Nervosa

$\mathrm{j}$ Weighted numbers; numbers of single diagnoses exceed numbers of aggregated groupings if comorbid.

$\mathrm{k}$ Weighted percentages and standard errors. 


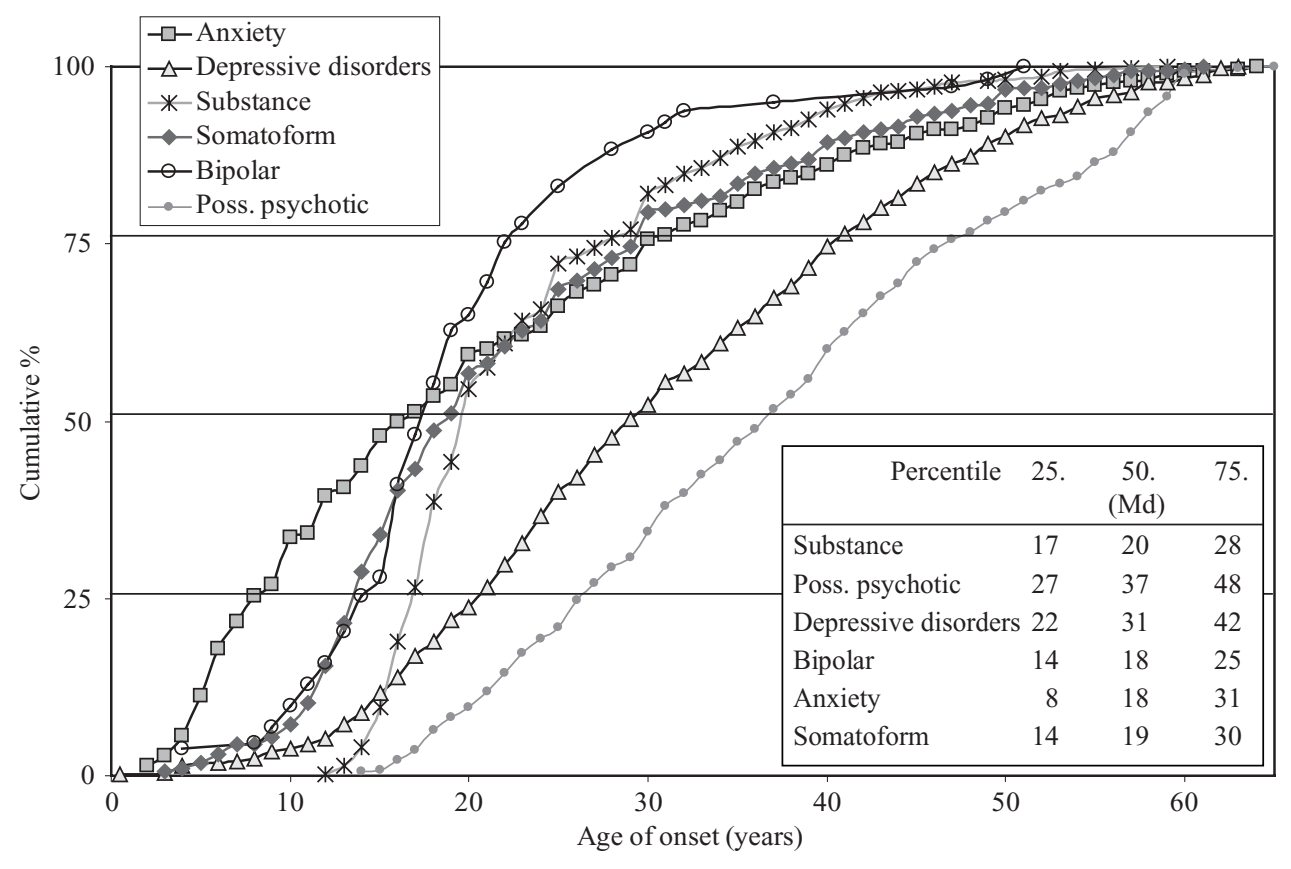

FIg. 1. Age of onset distributions.

\section{Co-morbidity}

Table 2 reveals rates for 12-month co-morbidity. $60.5 \%$ of the diagnosed subjects had a single diagnosis and $39.5 \%$ were co-morbid (4week: $36 \cdot 3 \%$; lifetime: $43 \cdot 1 \%$; not shown in Table 2). $10 \cdot 3 \%$ were highly co-morbid ( $>3$ disorders; 4-week: $10 \cdot 3 \%$; lifetime: $10 \cdot 5 \%$; not shown in Table 2). It has to be mentioned that these results on co-morbidity are crosssectional. Taking lifetime co-morbidity into account, the co-morbidity rate of respondents with a given 4-week diagnosis is shifted from $36 \%$ to $64 \%$ and for 12 -month diagnoses from $39 \%$ to $53 \%$ (not shown in Table 2 ).

Table 2, left section, shows proportions of 'pure' disorders and one/two/three or more additional diagnoses per disorder. A total of 2321 diagnoses were assigned to the 1301 respondents with at least one disorder. Pure disorders in the past 12 months were quite rare. Only 78612 -month diagnoses assigned were pure, whereas 1327 diagnoses were co-morbid (mean over all diagnoses: 66\%). Proportions of co-morbidity (at least one co-morbid condition) ranged from $44 \%$ for alcohol abuse/dependence to $94 \%$ for generalized anxiety disorder. The rate for highly co-morbid disorders ( $>3$ disorders) was lowest in substance use disorders $(12 \cdot 9-14 \cdot 4 \%)$ and highest in possible psychotic disorder $(40 \%)$, panic disorder $(49 \%)$, generalized anxiety disorder (53\%) and obsessive compulsive disorder $(63 \%)$.

We also determined the most frequent combinations of seven aggregated diagnostic groups [any substance use disorder, any possible psychotic disorder, any depressive disorder, any bipolar disorder, any anxiety disorder, any somatoform disorder, any eating disorder; 12month diagnoses] among the co-morbid cases $(n=514)$. Fifty-two different combinations out of the logically possible $127\left(2^{7}-1\right)$ occurred at least one time, among them five relatively frequent patterns that accounted for $60 \%$ of all co-morbid patterns: depression-anxiety $(19 \%)$, anxiety-somatoform (12\%), depressive-anxietysomatoform $(11 \%)$, anxiety-anxiety $(10 \%)$, and depressive-somatoform $(8 \%)$.

\section{Health care utilization}

Only $40 \%$ of respondents with at least one 12-month mental disorder received at least a 'minimal intervention' for their condition 
Table 2. Co-morbidity and health care utilization in subjects with at least one 12-month DSM-IV disorder ${ }^{\mathrm{a}}$

\begin{tabular}{|c|c|c|c|c|c|c|c|c|c|}
\hline \multirow[b]{2}{*}{ Disorders (DSM-IV) ${ }^{\mathrm{c}}$} & \multicolumn{4}{|c|}{ Proportions with } & \multicolumn{5}{|c|}{ Health care utilization: proportions of cases with at least minimal intervention ${ }^{\mathrm{b}}$} \\
\hline & $\begin{array}{c}\text { Pure } \\
\text { disorder } \\
\%\end{array}$ & $\begin{array}{c}\text { One } \\
\text { additional } \\
\text { diagnosis } \\
\%\end{array}$ & $\begin{array}{c}\text { Two } \\
\text { additional } \\
\text { diagnoses } \\
\%\end{array}$ & $\begin{array}{c}\text { Three or more } \\
\text { additional } \\
\text { diagnoses } \\
\%\end{array}$ & $\begin{array}{c}\text { Total } \\
\%\end{array}$ & $\begin{array}{c}\text { Pure } \\
\text { disorder } \\
\%\end{array}$ & $\begin{array}{c}\text { One } \\
\text { additional } \\
\text { diagnosis } \\
\%\end{array}$ & $\begin{array}{c}\text { Two } \\
\text { additional } \\
\text { diagnoses } \\
\%\end{array}$ & $\begin{array}{c}\text { Three or more } \\
\text { additional } \\
\text { diagnoses } \\
\%\end{array}$ \\
\hline $\begin{array}{l}\text { Any mental disorder due to general } \\
\text { medical condition }\end{array}$ & $18 \cdot 8$ & $38 \cdot 1$ & $13 \cdot 7$ & $29 \cdot 5$ & $59 \cdot 6$ & $58 \cdot 8$ & $60 \cdot 0$ & $76 \cdot 9$ & $51 \cdot 7$ \\
\hline Any substance disorder & $55 \cdot 1$ & $22 \cdot 8$ & $7 \cdot 8$ & $14 \cdot 3$ & $35 \cdot 5$ & $22 \cdot 9$ & $33 \cdot 6$ & $63 \cdot 0$ & $67 \cdot 2$ \\
\hline Alcohol abuse/dependence & $55 \cdot 7$ & $23 \cdot 3$ & $6 \cdot 7$ & $14 \cdot 4$ & $34 \cdot 4$ & $22 \cdot 3$ & $36 \cdot 2$ & $56 \cdot 7$ & $68 \cdot 1$ \\
\hline Any illicit substance abuse/dependence & $45 \cdot 3$ & $29 \cdot 0$ & $12 \cdot 9$ & $12 \cdot 9$ & $32 \cdot 7$ & $25 \cdot 2$ & $14 \cdot 3$ & $72 \cdot 5$ & $60 \cdot 9$ \\
\hline Possible psychotic disorder & $27 \cdot 1$ & $19 \cdot 8$ & $13 \cdot 3$ & $39 \cdot 9$ & $56 \cdot 6$ & $30 \cdot 3$ & $45 \cdot 1$ & $50 \cdot 1$ & $82 \cdot 4$ \\
\hline Any mood disorder & $38 \cdot 8$ & $21 \cdot 8$ & $15 \cdot 7$ & $23 \cdot 7$ & $52 \cdot 5$ & $41 \cdot 0$ & $40 \cdot 6$ & $61 \cdot 6$ & $76 \cdot 2$ \\
\hline Any depressive disorder & $39 \cdot 3$ & $20 \cdot 8$ & $15 \cdot 8$ & $24 \cdot 1$ & $53 \cdot 5$ & $41 \cdot 2$ & $42 \cdot 1$ & $62 \cdot 4$ & $77 \cdot 8$ \\
\hline Any bipolar disorder & $25 \cdot 7$ & $24 \cdot 6$ & $14 \cdot 9$ & $34 \cdot 9$ & $51 \cdot 8$ & $42 \cdot 2$ & $42 \cdot 4$ & $58 \cdot 8$ & $62 \cdot 4$ \\
\hline Any anxiety disorder & $37 \cdot 9$ & $26 \cdot 5$ & $14 \cdot 2$ & $21 \cdot 3$ & $47 \cdot 0$ & $27 \cdot 1$ & $46 \cdot 7$ & $57 \cdot 5$ & $75 \cdot 6$ \\
\hline Panic disorder & $11 \cdot 7$ & $26 \cdot 9$ & $12 \cdot 4$ & $49 \cdot 0$ & $75 \cdot 4$ & $54 \cdot 3$ & $51 \cdot 4$ & $79 \cdot 0$ & $92 \cdot 6$ \\
\hline Any phobia & $39 \cdot 8$ & $25 \cdot 4$ & $13 \cdot 7$ & $21 \cdot 2$ & $45 \cdot 3$ & $25 \cdot 2$ & $45 \cdot 7$ & $56 \cdot 4$ & $75 \cdot 7$ \\
\hline Generalized anxiety disorder & $6 \cdot 4$ & $15 \cdot 5$ & $24 \cdot 7$ & $53 \cdot 3$ & $68 \cdot 8$ & $55 \cdot 6$ & $69 \cdot 1$ & $56 \cdot 1$ & $76 \cdot 2$ \\
\hline Obsessive compulsive disorder & $14 \cdot 9$ & $12 \cdot 3$ & $9 \cdot 6$ & $63 \cdot 2$ & $68 \cdot 2$ & $20 \cdot 7$ & $66 \cdot 1$ & $39 \cdot 5$ & $84 \cdot 1$ \\
\hline Any somatoform disorder/syndrome & $45 \cdot 7$ & $21 \cdot 2$ & $14 \cdot 7$ & $18 \cdot 5$ & $42 \cdot 5$ & $27 \cdot 2$ & $42 \cdot 4$ & $53 \cdot 9$ & $71 \cdot 3$ \\
\hline SSI4.6 & $34 \cdot 2$ & $22 \cdot 1$ & $19 \cdot 2$ & $24 \cdot 5$ & $49 \cdot 6$ & $32 \cdot 9$ & $47 \cdot 7$ & $58 \cdot 9$ & $67 \cdot 1$ \\
\hline Pain disorder & $43 \cdot 5$ & $22 \cdot 0$ & $14 \cdot 7$ & $19 \cdot 7$ & $40 \cdot 0$ & $25 \cdot 0$ & $35 \cdot 6$ & $51 \cdot 7$ & $69 \cdot 6$ \\
\hline Any eating disorder & $34 \cdot 8$ & $21 \cdot 1$ & $30 \cdot 8$ & $13 \cdot 3$ & $47 \cdot 0$ & $49 \cdot 1$ & - & $85 \cdot 2$ & $27 \cdot 5$ \\
\hline Any mental disorder & $60 \cdot 5$ & $20 \cdot 3$ & $9 \cdot 0$ & $10 \cdot 3$ & $40 \cdot 5$ & $30 \cdot 6$ & $43 \cdot 5$ & $59 \cdot 6$ & $76 \cdot 1$ \\
\hline
\end{tabular}

a 2321 diagnoses were assigned to $n=1301$ respondents with at least one diagnosis out of $n=4181$ (total sample GHS-MHS).

b Health care utilization is presented in form of a variable 'at least minimal intervention', a combination of items asking for having ever been seeking treatment due to psychological, mental, addictive or psychosomatic problems, or been recommended by a doctor to do so. The so-defined overall treatment rate was \% in respondents with at least one diagnosis and \% in respondents with no diagnosis.

c For detailed information on included disorders see notes to Table 1. 
(having ever sought any kind of treatment due to mental health problems or having been recommended by a doctor to do so). It should be noted that if a co-morbid case was treated this treatment is shown for every co-morbid disorder, because in the present analysis it cannot be determined for which disorder(s) this person was treated; therefore numbers in Table 2 for specific disorders exceed the overall participant's treatment rate (i.e. 'any mental disorder'). Treatment rates increase fairly steadily by number of comorbid conditions. For example, in panic disorder, the most frequently treated disorder, $54 \%$ of the pure and $93 \%$ of the highly co-morbid cases report an at least minimal intervention. Substance use disorders - the less frequently treated disorders - show treatment rates from $23 \%$ (pure cases) to $67 \%$ (highly co-morbid cases). Co-morbidity (in a model with sex, age and three dummy variables for the number of diagnoses) had the same predictive value (area under the ROC curve $=0.72$ ) as the presence of the different diagnoses (in a model with sex, age and 12 dummies for the different diagnoses).

\section{Correlates of mental disorders}

Sociodemographic and health-related correlates of 12-month disorders are shown in Table 3 with odds ratios (OR) and confidence intervals (CI).

The significant sex differences have been mentioned above: women have a lower risk of having a substance use disorder (OR 0.2), but a higher risk of having a mood disorder (OR 2.0), an anxiety disorder (OR 2.4) or a somatoform disorder (OR 2.3). Their average number of diagnoses is about $70 \%$ higher than in men (not shown in Table 3). No sex differences were found in possible psychotic disorders.

Age effects played only a minor role, except for substance use disorders, where respondents older than 34 years of age had significantly fewer disorders than respondents aged 18-34 (35-49 years: OR 0.4; 50-65 years: OR $0 \cdot 3$ ).

Marital status is a significant correlate of all mental disorders (except for somatoform disorders): being never married, separated, divorced or widowed increases the probability of a diagnosis substantially (OR 1.5-2.9). The separated/divorced or widowed respondents have $80 \%$ more diagnoses than the married ones (singles: $50 \%$ more diagnoses; not shown in Table 3). Respondents from the lower social class have significantly higher prevalences of substance use disorders, mood disorders, and anxiety disorders compared with a higher social class (OR $0.5-0.8$ for medium or upper social class). With regard to somatoform disorders, only the upper social class has a lower prevalence rate than the low social class. Unemployed and retired people in particular have elevated risks for having a mental disorder (OR 1 $5-2 \cdot 3$, except for possible psychotic disorder, where associations were slightly insignificant). So the presented GHS-MHS data support the view that rates of most mental disorders decline with increasing social status. The social class index used (Winkler-Schicht Index; Winkler \& Stolzenberg, 1998) is calculated from information on education, current job status and household net income; differential data on education and employment status is also given in Table 3.

Concerning physical illness (assessed by MDs with a clinical structured computer assisted interview; Jacobi et al. 2002), poor health status is strongly associated with all mental disorders (OR 1.9-4.0).

\section{DISCUSSION}

\section{Design and sampling issues}

This paper presents prevalence estimates of the first nationwide German mental health survey conducted after the reunification of former East and West Germany and thus provides for the first time ever comprehensive data about the prevalence of mental disorders and their burden in Germany. The conduct of this survey as part of the regular national somatic health survey is a significant step forward to an integrated national health report system in Germany. The joint assessment of mental disorders and physical morbidities along with data on disability, work disability, service utilization and treatment within one modular study offers unique cross-comparisons between these domains that will be dealt with in subsequent steps of the analyses. Before highlighting the major findings a few comments with regard to design and methods will be given.

The high conditional response rate of almost $90 \%$ for the mental health supplement can be regarded as satisfactory in light of the non-response interview findings. Additional 
Table 3. Sociodemographic and health related correlates of major diagnostic subgroups and comorbidity (12-month $)^{\mathrm{a}}$

\begin{tabular}{|c|c|c|c|c|c|c|c|c|c|c|c|c|c|c|c|c|}
\hline & \multirow{3}{*}{$\begin{array}{c}\text { Distribution } \\
\text { of correlate } \\
\text { in sample } \\
\%\end{array}$} & \multicolumn{15}{|c|}{ 12-month prevalence if correlate present } \\
\hline & & \multicolumn{3}{|c|}{$\begin{array}{l}\text { Any substance } \\
\text { disorder }\end{array}$} & \multicolumn{3}{|c|}{$\begin{array}{c}\text { Any possible } \\
\text { psychotic disorder }\end{array}$} & \multicolumn{3}{|c|}{$\begin{array}{l}\text { Any mood } \\
\text { disorder }\end{array}$} & \multicolumn{3}{|c|}{$\begin{array}{l}\text { Any anxiety } \\
\text { disorder }\end{array}$} & \multicolumn{3}{|c|}{$\begin{array}{l}\text { Any somatoform } \\
\text { disorder }\end{array}$} \\
\hline & & $\%$ & $\mathrm{OR}^{\mathrm{b}}$ & $95 \% \mathrm{CI}$ & $\%$ & $\mathrm{OR}^{\mathrm{b}}$ & $95 \% \mathrm{CI}$ & $\%$ & $\mathrm{OR}^{\mathrm{b}}$ & $95 \% \mathrm{CI}$ & $\%$ & $\mathrm{OR}^{\mathrm{b}}$ & $95 \% \mathrm{CI}$ & $\%$ & $\mathrm{OR}^{\mathrm{b}}$ & $95 \% \mathrm{CI}$ \\
\hline \multicolumn{17}{|l|}{ Sex } \\
\hline Men & $50 \cdot 3$ & $7 \cdot 2$ & & & $2 \cdot 6$ & & & $8 \cdot 5$ & & & $9 \cdot 2$ & & & $7 \cdot 1$ & & \\
\hline Women & $49 \cdot 7$ & 1.7 & $0 \cdot 2^{*}$ & $0 \cdot 2-0 \cdot 3$ & $2 \cdot 6$ & $1 \cdot 0$ & $0 \cdot 7-1 \cdot 5$ & $15 \cdot 4$ & $2 \cdot 0^{*}$ & $1 \cdot 6-2 \cdot 4$ & $19 \cdot 8$ & $2 \cdot 4^{*}$ & $2 \cdot 0-3 \cdot 0$ & $15 \cdot 0$ & $2 \cdot 3^{*}$ & $1 \cdot 8-2 \cdot 8$ \\
\hline \multicolumn{17}{|l|}{ Age } \\
\hline $18-34$ & $34 \cdot 5$ & $7 \cdot 7$ & & & $2 \cdot 9$ & & & $11 \cdot 3$ & & & $13 \cdot 9$ & & & $10 \cdot 2$ & & \\
\hline $35-49$ & $34 \cdot 0$ & $3 \cdot 5$ & $0 \cdot 4 *$ & $0 \cdot 3-0 \cdot 6$ & $2 \cdot 6$ & $0 \cdot 9$ & $0 \cdot 5-1 \cdot 5$ & $12 \cdot 6$ & $1 \cdot 1$ & $0 \cdot 9-1 \cdot 4$ & $14 \cdot 6$ & $1 \cdot 1$ & $0 \cdot 9-1 \cdot 3$ & $11 \cdot 2$ & $1 \cdot 1$ & $0 \cdot 9-1 \cdot 4$ \\
\hline $50-65$ & $31 \cdot 6$ & $2 \cdot 1$ & $0 \cdot 3^{*}$ & $0 \cdot 2-0 \cdot 4$ & $2 \cdot 2$ & $0 \cdot 7$ & $0 \cdot 5-1 \cdot 2$ & $11 \cdot 8$ & $1 \cdot 0$ & $0 \cdot 8-1 \cdot 3$ & $14 \cdot 9$ & $1 \cdot 1$ & $0 \cdot 9-1 \cdot 3$ & $11 \cdot 7$ & $1 \cdot 2$ & $0 \cdot 9-1 \cdot 5$ \\
\hline \multicolumn{17}{|l|}{ Marital status } \\
\hline Married & $64 \cdot 1$ & $2 \cdot 7$ & & & $2 \cdot 0$ & & & $9 \cdot 8$ & & & $13 \cdot 2$ & & & $10 \cdot 6$ & & \\
\hline Single & $24 \cdot 9$ & $8 \cdot 8$ & $1.9 *$ & $1 \cdot 2-3 \cdot 1$ & $3 \cdot 6$ & $1 \cdot 8^{*}$ & $1 \cdot 1-2 \cdot 8$ & $13 \cdot 2$ & $1 \cdot 6^{*}$ & $1 \cdot 2-2 \cdot 3$ & $15 \cdot 1$ & $1 \cdot 4 *$ & $1 \cdot 1-1 \cdot 8$ & $9 \cdot 7$ & $1 \cdot 0$ & $0 \cdot 8-1 \cdot 4$ \\
\hline $\begin{array}{l}\text { Separated/divorced/ } \\
\text { widowed }\end{array}$ & $11 \cdot 0$ & $5 \cdot 6$ & $2 \cdot 9^{*}$ & $1 \cdot 8-4 \cdot 6$ & $3 \cdot 8$ & 1.9 & $0 \cdot 9-4 \cdot 1$ & $22 \cdot 6$ & $2 \cdot 5^{*}$ & $1 \cdot 9-3 \cdot 3$ & $20 \cdot 2$ & $1 \cdot 5^{*}$ & $1 \cdot 1-1 \cdot 9$ & $15 \cdot 0$ & $1 \cdot 3$ & $0 \cdot 9-1 \cdot 8$ \\
\hline \multicolumn{17}{|l|}{ Employment status ${ }^{\mathrm{c}}$} \\
\hline \multicolumn{17}{|l|}{ Employed } \\
\hline Full time & $49 \cdot 8$ & $4 \cdot 7$ & & & $2 \cdot 6$ & & & $9 \cdot 1$ & & & $10 \cdot 8$ & & & $9 \cdot 0$ & & \\
\hline $15-34 \mathrm{~h} /$ week & $8 \cdot 1$ & $2 \cdot 3$ & $1 \cdot 5$ & $0 \cdot 7-3 \cdot 3$ & 0.8 & $0 \cdot 3^{*}$ & $0 \cdot 1-0 \cdot 8$ & $11 \cdot 0$ & $0 \cdot 9$ & $0 \cdot 6-1 \cdot 3$ & $19 \cdot 9$ & $1 \cdot 3$ & $0 \cdot 9-1 \cdot 8$ & $15 \cdot 1$ & $1 \cdot 1$ & $0 \cdot 8-1 \cdot 6$ \\
\hline$<15 \mathrm{~h} /$ week & $4 \cdot 8$ & 0.5 & $0 \cdot 4$ & $0 \cdot 1-2 \cdot 6$ & $0 \cdot 5$ & $0 \cdot 2^{*}$ & $0 \cdot 1-0 \cdot 8$ & $14 \cdot 2$ & $1 \cdot 2$ & $0 \cdot 7-1 \cdot 9$ & $15 \cdot 8$ & $1 \cdot 0$ & $0 \cdot 6-1 \cdot 5$ & $9 \cdot 7$ & $0 \cdot 7$ & $0 \cdot 4-1 \cdot 2$ \\
\hline \multicolumn{17}{|l|}{ Not employed } \\
\hline Retired & $9 \cdot 7$ & $3 \cdot 0$ & 1.9 & $0 \cdot 9-4 \cdot 4$ & 1.6 & 0.6 & $0 \cdot 3-1 \cdot 6$ & $16 \cdot 3$ & $2 \cdot 1 *$ & $1 \cdot 4-3 \cdot 1$ & $17 \cdot 2$ & $1 \cdot 5^{*}$ & $1 \cdot 1-2 \cdot 2$ & $13 \cdot 8$ & $1 \cdot 4$ & $0 \cdot 9-2 \cdot 1$ \\
\hline School/student & $5 \cdot 7$ & $10 \cdot 3$ & 1.7 & $0 \cdot 9-3 \cdot 0$ & $3 \cdot 8$ & $1 \cdot 4$ & $0 \cdot 7-3 \cdot 1$ & $12 \cdot 1$ & $1 \cdot 2$ & $0 \cdot 7-1 \cdot 9$ & $12 \cdot 2$ & $1 \cdot 0$ & $0 \cdot 6-1 \cdot 6$ & $7 \cdot 2$ & $0 \cdot 7$ & $0 \cdot 4-1 \cdot 2$ \\
\hline Unemployed & $6 \cdot 5$ & $6 \cdot 3$ & $2 \cdot 0^{*}$ & $1 \cdot 2-3 \cdot 6$ & $3 \cdot 1$ & $1 \cdot 2$ & $0 \cdot 5-2 \cdot 7$ & $20 \cdot 0$ & $2 \cdot 3^{*}$ & $1 \cdot 6-3 \cdot 2$ & $23 \cdot 2$ & $2 \cdot 2 *$ & $1 \cdot 6-3 \cdot 0$ & $16 \cdot 0$ & $1 \cdot 6^{*}$ & $1 \cdot 1-2 \cdot 4$ \\
\hline Homemaker & $7 \cdot 2$ & $0 \cdot 7$ & $0 \cdot 6$ & $0 \cdot 1-2 \cdot 6$ & $4 \cdot 1$ & 1.6 & $0 \cdot 7-3 \cdot 4$ & $17 \cdot 8$ & $1 \cdot 5^{*}$ & $1 \cdot 0-2 \cdot 2$ & $19 \cdot 4$ & $1 \cdot 2$ & $0 \cdot 8-1 \cdot 7$ & $11 \cdot 1$ & $0 \cdot 7$ & $0 \cdot 5-1 \cdot 1$ \\
\hline \multicolumn{17}{|l|}{ Social class ${ }^{\mathrm{d}}$} \\
\hline Low & $19 \cdot 1$ & $6 \cdot 6$ & & & $3 \cdot 5$ & & & $16 \cdot 4$ & & & $18 \cdot 6$ & & & $13 \cdot 5$ & & \\
\hline Medium & $57 \cdot 6$ & $4 \cdot 2$ & $0 \cdot 6^{*}$ & $0 \cdot 4-0 \cdot 9$ & $2 \cdot 4$ & $0 \cdot 7$ & $0 \cdot 4-1 \cdot 1$ & $12 \cdot 0$ & $0 \cdot 7 *$ & $0 \cdot 6-0 \cdot 9$ & $14 \cdot 4$ & $0 \cdot 8^{*}$ & $0 \cdot 6-0 \cdot 9$ & $10 \cdot 6$ & $0 \cdot 8$ & $0 \cdot 6-1 \cdot 0$ \\
\hline High & $23 \cdot 3$ & $3 \cdot 7$ & $0 \cdot 6^{*}$ & $0 \cdot 4-0 \cdot 9$ & $2 \cdot 2$ & 0.6 & $0 \cdot 3-1 \cdot 2$ & $8 \cdot 8$ & $0 \cdot 5^{*}$ & $0 \cdot 4-0 \cdot 7$ & $11 \cdot 3$ & $0 \cdot 6^{*}$ & $0 \cdot 4-0 \cdot 8$ & $9 \cdot 3$ & $0 \cdot 7 *$ & $0 \cdot 5-0 \cdot 9$ \\
\hline \multicolumn{17}{|l|}{ Somatic health status ${ }^{\mathrm{e}}$} \\
\hline Good & $29 \cdot 3$ & 4.9 & & & 1.7 & & & $7 \cdot 5$ & & & $10 \cdot 1$ & & & $5 \cdot 3$ & & \\
\hline Medium & $45 \cdot 4$ & $4 \cdot 7$ & $1 \cdot 4$ & $0 \cdot 9-2 \cdot 1$ & $2 \cdot 5$ & 1.6 & $0 \cdot 9-3 \cdot 0$ & $12 \cdot 0$ & $1 \cdot 6^{*}$ & $1 \cdot 2-2 \cdot 1$ & $12 \cdot 5$ & $1 \cdot 2$ & $0 \cdot 9-1 \cdot 5$ & $10 \cdot 6$ & $2 \cdot 1^{*}$ & $1 \cdot 5-2 \cdot 8$ \\
\hline Poor & $24 \cdot 7$ & $3 \cdot 6$ & $1.9 *$ & $1 \cdot 2-3 \cdot 2$ & $3 \cdot 7$ & $2 \cdot 9 *$ & $1 \cdot 7-5 \cdot 0$ & $17 \cdot 2$ & $2 \cdot 5^{*}$ & $1 \cdot 8-3 \cdot 4$ & $23 \cdot 2$ & $2 \cdot 5^{*}$ & $1 \cdot 9-3 \cdot 3$ & $18 \cdot 8$ & $4 \cdot 0^{*}$ & $2 \cdot 8-5 \cdot 6$ \\
\hline
\end{tabular}

a GHS-MHS, weighted data, $n=4181$.

b Odds ratios (OR) from logistic regression and $95 \%$ confidence intervals $(\mathrm{CI})$, controlled for age and sex; reference groups: not having the disorder under consideration; * $p<0 \cdot 05$.

c 'Other' employment status (e.g. maternity leave, military or civil service) excluded from analyses.

d Index of social class (Winkler \& Stolzenberg, 1998) derived from information on education, income and current (job) position.

e Crude indicator derived from the amount of somatic diseases assessed in the GHS-CS. Good: no somatic diseases within past 12 months; medium: $1-2$ somatic diseases within past 12 months; poor: 3 or more somatic diseases within past 12 months. 
checks for limited representativeness as compared to the total population provided no evidence for any major systematic sample bias (Jacobi et al. 2002).

Retrospective data on age of onset are notoriously unreliable, but may be useful in the absence of other data. In the present crosssectional study, probes were embedded in the interviews in order to reduce recall bias. Yet, this cannot rule out systematic under-reporting due to embarrassment or fear of discrimination (Kessler, 2000).

We want to emphasize that this first prevalence publication reports predominantly diagnostically aggregated data. Findings on specific subtypes and disorders will be dealt with in separate follow-up publications.

Particular strengths of the study are as follows. (1) It is largely built on standardized assessment instruments with an established reliability allowing for direct comparisons with other studies. (2) It includes a considerably larger number of specific mental disorders according to DSM-IV criteria than previous studies. (3) Unlike other community surveys using the same instrument, we focused on 12-month and 4-week estimates of symptoms and diagnoses of mental disorders to increase precision and reliability and to obtain straightforward 12-month symptom counts. Lifetime disorders not present in the 12month were only assessed for mood, psychotic and selected other disorders once the 12-month assessment was completed. Not relying only on recency information of lifetime diagnoses when determining 12-month diagnoses provides greater accuracy. Yet, the 12-month orientation means that those without a current disorder are less likely to report a lifetime disorder, resulting in an over-representation of chronic cases with an increased likelihood of early onset that has to be considered in future analyses dealing with lifetime diagnoses. (4) Interviews were conducted by clinically trained interviewers, since the use of lay interviewers has often been mentioned as a limitation of previous large-scale nationwide surveys. This also allowed for the inclusion of both psychotic syndromes, as well as general medical factors.

\section{Prevalence}

Turning to the main findings, the study confirms that mental disorders are highly prevalent, affecting a substantial proportion of the adult German population aged $18-65$, with a total 12 -month prevalence of $31 \%$, a 4 -week prevalence of $20 \%$ and a lifetime prevalence of $43 \%$.

The GHS-MHS covers a relatively wide range of more than 60 mental disorders, allowing for a high degree of specification within seven diagnostic groupings.

It is important to note that the rates of morbidity shown in this study are not easily comparable to findings from other community studies due to different diagnostic criteria (ICD-10 v. DSM-III-R v. DSM-IV), different age ranges (e.g. higher prevalences of substance use disorders in younger samples), and in particular due to the inclusion of different diagnoses. Some studies included additional diagnoses (e.g. post-traumatic stress disorder and antisocial personality in the NCS) or a more restricted range of disorders (e.g. neglect of somatoform disorders in most previous studies; Faravelli et al. 1997). This selection has some effect on the total prevalence estimated. Regarding the frequency of DSM-IV-disorders in our study, as an example, the exclusion of somatoform disorders lowers the overall lifetime prevalence from about $43 \%$ to $37 \%$ (mostly due to 200 respondents with a pure somatoform pain disorder).

Nevertheless our results show a relatively strong concordance with most comparable studies (e.g. those reported by Kessler et al. 1994; Bijl et al. 1998; Andrade et al. 2000; Andrews et al. 2001). The overall 12-month prevalence in the GHS-MHS is $31 \cdot 1 \%$ for the reported seven diagnostic DSM-IV groupings (and 25.7\% when excluding somatoform disorders, eating disorders and mental disorders due to a general medical condition which were not assessed in most other studies). These rates are comparable to the USA $(29.5 \%)$, The Netherlands $(23.2 \%)$, most of the other ICPE surveys (Brazil, Canada, Mexico, Turkey; 8.4-24.4\%), and Australia (20.3\%).

Rates for anxiety and mood disorders (14.5\% and $11.9 \%$ ) are also relatively similar to the USA $(17 \cdot 2 \%$ and $11.3 \%)$, slightly higher than in The Netherlands $(12.4 \%$ and $7.6 \%)$ and most of the other ICPE surveys $(4 \cdot 0-12 \cdot 4 \%$ and $4 \cdot 2-7 \cdot 1 \%)$, whereas in Australia remarkably lower rates were found $(5 \cdot 6 \%$ and $6 \cdot 6 \%)$. 
Substance use disorders were diagnosed less often $(4.5 \%$, with the vast majority of alcohol related disorders) than in the USA $(11.3 \%)$, The Netherlands $(8.9 \%)$, most other ICPE surveys $(0 \cdot 0-10 \cdot 5 \%)$ and Australia $(7 \cdot 9 \%)$. As mentioned above, when comparing these rates internationally it is important to take into account differences in the instruments (e.g. the UM-CIDI in the NCS is particularly sensitive; Kessler et al. 2000), and age of the sample (e.g. including only age 18-54 as in the NCS the GHS-MHS prevalence of any substance use disorder is shifted to $5 \cdot 2 \%$ ). Yet, the low prevalence is surprising since alcohol consumption, according to the WHO world drink trends reports, is comparatively high in Germany. An explanation could be the fact that diagnostic criteria focus more on (culturally and socially determined) inadequacy of drinking behaviour than on absolute quantity of consumption. Therefore, as an example, it is easier to qualify for alcohol abuse in the USA where the consumption of 1.5 litres of beer is labelled as 'binge drinking' compared to wide parts of the German society, where this amount is consumed several times a week without further negative social consequences. In particular, young people are more likely to receive a diagnosis of alcohol abuse in the USA where drinking in public is illegal until the age of 21 (Germany: 16 years).

\section{Age of onset/persistence}

The GHS-MHS findings also provide some further evidence that most mental disorders begin early. The median of the reported first onset of anxiety, somatoform, bipolar mood and substance use disorders is below 20 years of age. The median of depressive disorders and possible psychotic disorders is in young adulthood ( 31 v. 37 years), and only about $25 \%$ of the affected subjects develop their first disorder after their early 40s. The different characteristics match the average shapes of the cross-national ICPE-results (Andrade et al. 2000). Data on age of first onset were gathered retrospectively, and information on the course of a disorder between first onset and recent manifestation was not assessed. Since we have no longitudinal data on these issues, interpretations from this study on course of disorders, longitudinal comorbidity relevant for aetiological aspects of mental disorders, or persistence are definitely limited. However, there are some clues that might be interpreted as indirect estimates of the chronicity of mental disorders. The average time between reported age of onset and age of the participants with the respective current diagnosis at the interview ranged from 10 to 20 years. Three quarters of all lifetime diagnoses were still present in the last 12 months; the prevalence ratios between time frames (4-week 12-month; 4-week lifetime; 12-month lifetime) suggest that mental disorders are often stable and call into question older statements that mental disorders are characterized by high remission rates.

\section{Co-morbidity}

We also confirmed that co-morbidity is a common phenomenon in almost all mental disorders. By the way, this also appears to be true for co-morbidity among somatic illnesses. Since the patterns of co-morbidity are of a very complex nature due to the coverage of the high number of included disorders (Wittchen, $1996 a, b)$, results on co-morbidity were presented in two relatively crude ways: number of disorders per participant and amount of comorbidity per disorder. Regarding again the 12-month frame, about $40 \%$ of the diagnosed participants have more than one single disorder. In this context, it should be noted that co-morbidity rates depend heavily on the definition of co-morbidity. From the perspective of disorders, a total of 2321 diagnoses were assigned to 1301 subjects. All disorders were highly to extremely co-morbid, ranging from $44 \%$ (alcohol abuse/ dependence) to $88 \%$ (panic disorders) and $94 \%$ (generalized anxiety disorder).

\section{Health care utilization}

Disregarding the noteworthy differences in base rate probabilities of specific diagnoses, it is fair to estimate that only about one third of pure disorders receive at least a minimal health care intervention, opposed to almost three out of four highly co-morbid subjects. For each disorder, the presence of co-morbid disorders is associated with a substantially higher rate of help seeking. This is consistent with previous findings from the USA and Canada (Kessler et al. 1994; Merikangas et al. 1996). This 
replication in a German sample is remarkable, yet discouraging, as we expected a higher treatment rate due to the relatively well established German mental health care system. Unlike the USA and also to a lesser degree Canada, the German health care system is characterized by a fairly dense network of medical and psychological services that all provide mental health care (including drugs and psychotherapy) free of charge and without any major limitation of access and treatment duration. Patients can even bypass primary care physicians that usually maintain a core gatekeeper function for specialized mental health care in most other countries. Although we have no data in this study on speed of treatment entry after developing the first mental disorder, it appears that treatment is only likely whenever the patient already has developed complex co-morbid patterns. Future analyses should examine whether earlier targeted treatment reduces co-morbidity and associated burden in terms of disability and reduced quality of life.

\section{Correlates}

As in all comparable studies, women report higher mental morbidity than men except for substance use disorders (where men are diagnosed four times as frequently as women), and possible psychotic disorders (where no sex differences were found). There have been no significant age effects on prevalence except for substance use disorders (more frequent in younger age).

Also, other correlates of mental disorders are in line with existing knowledge (lower risk for married, and for higher social class). However, findings of this kind are limited, since risk factors of this sort are either not modifiable or not specific enough for developing intervention strategies (Kessler, 2000). Further analyses will focus on the role of co-morbidity in the prediction of (further) mental disorders, impairment and reduced quality of life since co-morbidity may be a modifiable risk factor for illness course, future impairment and quality of life that can be more easily targeted than sociodemographic correlates of mental morbidity. Of special interest in this context and suggesting need for further research are the presented high associations of mental disorders and poor somatic health status.

\section{Conclusions for future analyses and studies}

Further analyses will determine the impact of mental disorders both on individual suffering and health related quality of life, and on direct and indirect societal costs of disorders (health care costs, loss of work productivity). Future studies should be designed to investigate modifiable risk factors of illness onset and course in greater detail, including developmental and genetic aspects (Wittchen, 1996 b; Kessler, 2000). Further research on determinants of help seeking and health care delivery is also needed since pathways to adequate mental health care seem not only to be disorder-specific or determined by severity of a condition, but also by other factors (e.g. knowledge about mental disorders in patients or in primary care physicians, or limitations within a health care system).

Kessler et al. (2002) suggest, on the basis of findings from the World Mental Health surveys on the distinction of generalized anxiety disorder from depression, that more comprehensive analyses within the complex area of co-morbidity are strongly needed in psychiatric epidemiology. A better understanding of patterns of comorbidity is relevant for diagnostic and nosological reasons and aetiological research on mental disorders on the one hand, and for the improvement of prevention and treatment strategies on the other hand, since co-morbidity affects onset, course and severity of the single disorders involved. In particular investigations on comorbidity with somatic conditions will enhance knowledge on health and illness as comprehensive concepts.

\section{ACKNOWLEDGEMENTS}

This study was supported by grant $01 \mathrm{EH} 970 / 8$ (German Federal Ministry of Research, Education and Science; BMBF). Reported data on mental disorders were assessed in the Mental Health Supplement of the German Health Survey (GHS-MHS), conducted by the MaxPlanck-Institute of Psychiatry, Munich. Principal investigator was Prof. Dr Hans-Ulrich Wittchen. Reported sociodemographic and somatic health status variables come from the Core Survey (GHS-CS), conducted by the Robert Koch-Institute, Berlin. Principal investigators of the GHS-CS were Dr Bärbel-Maria Kurth and Dr Wolfgang Thefeld. 


\section{NOTE}

Data of this study are available as Public Use File from the first author (manual and variable description in German language): Dr Frank Jacobi, Institute of Clinical Psychology and Psychotherapy, Chemnitzer Str. 46, D-01187 Dresden, Germany; e-mail: jacobi@psychologie.tu-dresden.de

For further information about the Core Survey (GHS-CS) and its Public Use File contact the Robert Koch-Institute, Dr Heribert Stolzenberg, Nordufer 20, D-13353 Berlin, Germany; e-mail: stolzenbergh@rki.de

\section{REFERENCES}

Andrade, L., Caraveo-Anduaga, J. J., Berglund, P., Bijl, R. V., Kessler, R. C., Demler, O., Walters, E., Kylyc, C., Offord, D., Üstün, T. B. \& Wittchen, H.-U. (2000). Cross-national comparisons of the prevalences and correlates of mental disorders. Bulletin of the World Health Organization 78, 413-426.

Andrews, G., Henderson, S. \& Hall, W. (2001). Prevalence, co-morbidity, disability and service utilisation. Overview of the Australian National Mental Health Survey. British Journal of Psychiatry 178, 145-153.

APA (1980). Diagnostic and Statistical Manual of Mental Disorders, 3rd edn. American Psychiatric Press: Washington, DC.

APA (1987). Diagnostic and Statistical Manual of Mental Disorders, 3rd edn, revised. American Psychiatric Press: Washington, DC

APA (1994). Diagnostic and Statistical Manual of Mental Disorders, 4th edn. American Psychiatric Press: Washington, DC.

Bellach, B.-M., Knopf, H. \& Thefeld, W. (1998). Der BundesGesundheitssurvey 1997/98. Gesundheitswesen 1998 60, 59-68.

Bijl, R. V. \& Ravelli, A. (2000). Current and residual functional disability associated with psychopathology: findings from The Netherlands Mental Health Survey and Incidence Study (NEMESIS). Psychological Medicine 30, 657-668.

Bijl, R. V., Ravelli, A. \& van Zessen, G. (1998). Prevalence of psychiatric disorder in the general population: results of The Netherlands Mental Health Survey and Incidence Study (NEMESIS). Social Psychiatry and Psychiatric Epidemiology 33, $587-595$.

Binder, D. A. (1983). On the variances of asymptotically normal estimators from complex surveys. International Statistical Review 51, 279-292.

Brugha, T. S., Bebbington, P. E., Jenkins, R., Meltzer, H., Taub, N. A., Janas, M. \& Vernon, J. (1999). Cross validation of a general population survey diagnostic interview: a comparison of CIS-R with SCAN ICD-10 diagnostic categories. Psychological Medicine 29, 1029-1042.

Carter, R. M., Wittchen, H.-U., Pfister, H. \& Kessler, R. C. (2001). One-year prevalence of subthreshold and threshold DSM-IV generalized anxiety disorder in a nationally representative sample. Depression and Anxiety 13, 78-88.

Escobar, J. I., Rubio Stipec, M., Canino, G. \& Karno, M. (1989). Somatic Symptom Index (SSI): a new and abridged somatization construct: prevalence and epidemiological correlates in two large community samples. Journal of Nervous and Mental Disease 177, $140-146$.

Faravelli, C., Salvatori, S., Galassi, F. \& Aiazzi, L. (1997). Epidemiology of somatoform disorders: a community survey in Florence. Social Psychiatry \& Psychiatric Epidemiology 32, 24-29.
Hagnell, O. (1970). The incidence and duration of episodes of mental illness in a total population. In Psychiatric Epidemiology (ed. E. H. Hare and J. K. Wing), pp. 213-224. Oxford University Press: London.

Jacobi, F., Wittchen, H.-U., Hölting, C., Sommer, S., Lieb, R., Höfler, M. \& Pfister, H. (2002). Estimating the prevalence of mental and somatic disorders in the community: aims and methods of the German National Health Interview and Examination Survey. International Journal of Methods in Psychiatric Research 11, $1-18$.

Jenkins, R., Lewis, G., Bebbington, P., Brugha, T., Farrell, M., Gill, B. \& Meltzer, H. (1997). The National Psychiatric Morbidity Surveys of Great Britain - initial findings from the household survey. Psychological Medicine 27, 775-789.

Kessler, R. C. (2000). Psychiatric epidemiology: selected recent and advances and future directions. Bulletin of the World Health Organization 78, 464-474.

Kessler, R. C., Berglund, P. A., DeWit, D. J., Üstün, T. B., Wang, P. S. \& Wittchen, H.-U. (2002). Distinguishing generalized anxiety disorder from major depression: prevalence and impairment from current pure and co-morbid disorders in the US and Ontario. International Journal of Methods in Psychiatric Research 11, 99-111.

Kessler, R. C., Greenberg, P. E., Mickelson, K. D., Meneades, L. M. \& Wang, P.S. (2001). The effects of chronic medical conditions on work loss and work cutback. Journal of Occupational and Environmental Medicine 43, 218-225.

Kessler, R. C., McGonagle, K. A., Zhao, S., Nelson, C. B., Hughes, M., Eshleman, S., Wittchen, H.-U. \& Kendler, K. S. (1994). Lifetime and 12-month prevalence of DSM-III-R psychiatric disorders in the United States: results from the National Comorbidity Survey. Archives of General Psychiatry 51, 8-19.

Kessler, R. C., Wittchen, H.-U., Abelson, J. \& Zhao, S. (2000). Methodological issues in assessing psychiatric disorders with selfreports. In The Science of Self-report: Implications for Research and Practice (ed. A. A. Stone et al.), pp. 229-255. Lawrence Erlbaum Associates: Mahwah, NJ, USA.

Knäuper, B. \& Wittchen, H.-U. (1994). Diagnosing major depression in the elderly: evidence for response bias in standardized diagnostic interviews? Journal of Psychiatric Research 28, 147-164.

Koran, L. M., Thienemann, M. L. \& Davenport, R. (1996). Quality of life for patients with obsessive compulsive disorder. American Journal of Psychiatry 153, 783-788.

Lachner, G., Wittchen, H.-U., Perkonigg, A., Holly, A., Schuster, P., Wunderlich, U., Türk, D., Garczynski, E. \& Pfister, H. (1998). Structure, content and reliability of the Munich-Composite International Diagnostic Interview (M-CIDI). Substance use sections. European Addiction Research 4, 28-41.

Merikangas, K. R., Angst, J., Eaton, W. W., Canino, G. J., RubioStipec, M., Wacker, H., Wittchen, H.-U., Andrade, L., Essau, C. A., Kraemer, H., Robins, L. \& Kupfer, D. J. (1996). Comorbidity and boundaries of affective disorders with anxiety disorders and substance misuse: results of an international task force. British Journal of Psychiatry 168 (Suppl. 30), 58-67.

Murray, C. J. \& Lopez, A. D. (1996). Evidence-based health policy - lessons from the global burden of disease study. Science 274, 740-743.

Ormel, J., Von Korff, M., Van den Brink, W., Katon, W., Brilman, E. \& Oldehinkel, T. (1993). Depression, anxiety and social disability show synchrony of change in primary care patients. American Journal of Public Health 83, 385-390.

Pyne, J. M., Patterson, T. L., Kaplan, R. M., Gillin, J. C., Koch, W. L. \& Grant, I. (1997). Assessment of the quality of life of patients with major depression. Psychiatric Services 48, 224-230.

Reed, V., Gander, F., Pfister, H., Steiger, A., Trenkwalder, C., Sonntag, H., Hundt, W. \& Wittchen, H.-U. (1998). To what degree the Composite International Diagnostic Interview (CIDI) correctly identifies DSM-IV disorders? Testing validity issues in a clinical sample. International Journal of Methods in Psychiatric Research 7, 142-155. 
Regier, D. A., Myers, J. K., Kramer, M., Robins, L. N., Blazer, D. G., Hough, R. L., Eaton, W. W. \& Locke, B. Z. (1984). The NIMH Epidemiologic Catchment Area program. Historical context, major objectives, and study population characteristics. Archives of General Psychiatry 41, 934-941.

Robins, N. L., et al. (1981). National Institute of Mental Health Diagnostic Interview Schedule: its history, characteristics and validity. Archives of General Psychiatry 38, 381-389.

Robins, N. L. \& Regier, D. A. (1990). Psychiatric Disorders in America: the Epidemiologic Catchment Area Study. Book News: Portland, Oregon.

Royall, R. M. (1986). Model robust confidence intervals using maximum likelihood estimators. International Statistical Review $\mathbf{5 4}, 221-226$.

StataCorp. (2001). Stata Statistical Software: Release 7.0 (Version College-Station). Stata Corporation: Texas, USA

Thefeld, W., Stolzenberg, H. \& Bellach, B. M. (1999). German National Health Interview and Examination Survey: response, composition of participants, and analysis of non-respondents. Gesundheitswesen 61, 57-61.

Weissman, M. M. \& The Cross National Collaborative Group (1993). Cross-National Epidemiologic Analyses (report): Upjohn Company.

Weissman, M. M., Bland, R. C., Canino, G. J., Faravelli, C., Greenwald, S., Hwu, H.-G., Joyce, P. R., Karam, E. G., Lee, C.-K., Lellouch, J., Lépine, J.-P., Newman, S. C., Rubio-Stipec, M., Wells, J. E., Wickramaratne, P. J., Wittchen, H.-U. \& Yeh, E.-K. (1996). Cross-national epidemiology of major depression and bipolar disorder. Journal of the American Medical Association 276, 293-299.

Wells, K. B., Stewart, A., Hays, R. D., Burnam, M. A., Rogers, W., Daniels, M., Berry, S., Greenfield, S. \& Ware, J. (1989). The functioning and well-being of depressed patients: results from the Medical Outcomes Study. Journal of the American Medical Association 262, 914-919.

Winkler, J. \& Stolzenberg, H. (1998). Der Sozialschichtindex im Bundes-Gesundheitssurvey. Das Gesundheitswesen 60, 21-26.

Wittchen, H.-U. (1988). Natural course and spontaneous remissions of untreated anxiety disorders - results of the Munich Follow-up
Study (MFS). In Panic and Phobias 2. Treatments and Variables Affecting Course and Outcome (ed. I. Hand and H.-U. Wittchen), pp. 3-17. Springer: Berlin.

Wittchen, H.-U. (1994). Reliability and validity studies of the WHOComposite International Diagnostic Interview (CIDI): a critical review. Journal of Psychiatric Research 28, 57-84.

Wittchen, H.-U. (1996a). What is co-morbidity - fact or artefact? British Journal of Psychiatry 168 (Suppl. 30), 7-8.

Wittchen, H.-U. (1996b). Critical issues in the evaluation of comorbidity of psychiatric disorders. British Journal of Psychiatry 168 (Suppl. 30), 9-16.

Wittchen, H.-U., Müller, N. \& Storz, S. (1998). Psychische Störungen: Häufigkeit, psychosoziale Beeinträchtigungen und Zusammenhänge mit körperlichen Erkrankungen. Gesundheitswesen 60, 85-100.

Wittchen, H.-U. \& Pfister, H. (1997). DIA-X-Interviews: Manual für Screening-Verfahren und Interview; Interviewheft Längsschnittuntersuchung (DIA-X-Lifetime); Ergänzungsheft (DIA-X-Lifetime) Interviewheft Querschnittuntersuchung (DIA-X-12 Monate), Ergänzungsheft (DIA-X-12Monate); PC-Programm zur Durchführung des Interviews (Längs- und Querschnittuntersuchung) Auswertungsprogramm. Swets \& Zeitlinger: Frankfurt.

Wittchen, H.-U., Üstün, T. B. \& Kessler, R. C. (1999a). Editorial. Diagnosing mental disorders in the community. A difference that matters? Psychological Medicine 29, 1021-1027.

Wittchen, H. U., Höfler, M., Gander, F., Pfister, H., Storz, S., Üstün, T. B., Müller, N. \& Kessler, R. C. (1999b). Screening for mental disorders: performance of the Composite International Diagnostic-Screener (CID-S). International Journal of Methods in Psychiatric Research 8: 59-70.

Woodruff, R.S. (1971). A simple method of approximating the variance of a complicated estimate. Journal of the American Statistical Association 66, 411-414.

WHO (1993). Tenth Revision of the International Classification of Diseases, Chapter V $(F)$ : Mental and Behavioral Disorders. Clinical Descriptions and Diagnostic Guidelines. World Health Organization: Geneva.

WHO (1997). Composite International Diagnostic Interview (CIDI, Version 2.1). World Health Organization: Geneva. 